\title{
Boundedness of fractional oscillatory integral operators and their commutators on generalized Morrey spaces
}

\section{Ahmet Eroglu*}

"Correspondence:

aeroglu@nigde.edu.tr Department of Mathematics, Nigde University, Nigde, Turkey

\begin{abstract}
In this paper, it is proved that both oscillatory integral operators and fractional oscillatory integral operators are bounded on generalized Morrey spaces $M_{p, \varphi}$. The corresponding commutators generated by $\mathrm{BMO}$ functions are also considered. MSC: Primary 42B20; 42B25; 42B35
\end{abstract}

Keywords: generalized Morrey space; oscillatory integral; commutator; BMO spaces

\section{Introduction and main results}

The classical Morrey spaces, were introduced by Morrey [1] in 1938, have been studied intensively by various authors and together with weighted Lebesgue spaces play an important role in the theory of partial differential equations; they appeared to be quite useful in the study of local behavior of the solutions of elliptic differential equations and describe local regularity more precisely than Lebesgue spaces.

Morrey spaces $\mathcal{M}_{p, \lambda}\left(\mathbb{R}^{n}\right)$ are defined as the set of all functions $f \in L_{p}\left(\mathbb{R}^{n}\right)$ such that

$$
\|f\|_{\mathcal{M}_{p, \lambda}} \equiv\|f\|_{\mathcal{M}_{p, \lambda}\left(\mathbb{R}^{n}\right)}=\sup _{x, r>0} r^{-\frac{\lambda}{p}}\|f\|_{L_{p}(B(x, r))}<\infty .
$$

Under this definition, $\mathcal{M}_{p, \lambda}\left(\mathbb{R}^{n}\right)$ becomes a Banach space; for $\lambda=0$, it coincides with $L_{p}\left(\mathbb{R}^{n}\right)$ and for $\lambda=1$ with $L_{\infty}\left(\mathbb{R}^{n}\right)$.

We also denote by $W \mathcal{M}_{p, \lambda}$ the weak Morrey space of all functions $f \in W L_{p}^{\text {loc }}\left(\mathbb{R}^{n}\right)$ for which

$$
\|f\|_{W \mathcal{M}_{p, \lambda}} \equiv\|f\|_{W \mathcal{M}_{p, \lambda}\left(\mathbb{R}^{n}\right)}=\sup _{x \in \mathbb{R}^{n}, r>0} r^{-\frac{\lambda}{p}}\|f\|_{W L_{p}(B(x, r))}<\infty,
$$

where $W L_{p}$ denotes the weak $L_{p}$-space.

Definition 1 Let $\varphi(x, r)$ be a positive measurable function on $\mathbb{R}^{n} \times(0, \infty)$ and $1 \leq p<\infty$. We denote by $M_{p, \varphi} \equiv M_{p, \varphi}\left(\mathbb{R}^{n}\right)$ the generalized Morrey space, the space of all functions $f \in L_{p}^{\text {loc }}\left(\mathbb{R}^{n}\right)$ with finite quasinorm

$$
\|f\|_{M_{p, \varphi}} \equiv\|f\|_{M_{p, \varphi}\left(\mathbb{R}^{n}\right)}=\sup _{x \in \mathbb{R}^{n}, r>0} \varphi(x, r)^{-1}|B(x, r)|^{-\frac{1}{p}}\|f\|_{L_{p}(B(x, r))} .
$$

o 2013 Eroglu; licensee Springer. This is an Open Access article distributed under the terms of the Creative Commons Attribution License (http://creativecommons.org/licenses/by/2.0), which permits unrestricted use, distribution, and reproduction in any medium, provided the original work is properly cited. 
Also, by $W M_{p, \varphi} \equiv W M_{p, \varphi}\left(\mathbb{R}^{n}\right)$, we denote the weak generalized Morrey space of all functions $f \in W L_{p}^{\text {loc }}\left(\mathbb{R}^{n}\right)$ for which

$$
\|f\|_{W M_{p, \varphi}} \equiv\|f\|_{W M_{p, \varphi}\left(\mathbb{R}^{n}\right)}=\sup _{x \in \mathbb{R}^{n}, r>0} \varphi(x, r)^{-1}|B(x, r)|^{-\frac{1}{p}}\|f\|_{W L_{p}(B(x, r))}<\infty .
$$

According to this definition, we recover the spaces $M_{p, \lambda}$ and $W M_{p, \lambda}$ under the choice $\varphi(x, r)=r^{\frac{\lambda-n}{p}}:$

$$
\begin{aligned}
& \left.M_{p, \varphi}\right|_{\varphi(x, r)=r \frac{\lambda-n}{p}}=M_{p, \lambda}, \\
& \left.W M_{p, \varphi}\right|_{\varphi(x, r)=r} \frac{\lambda-n}{p}=W M_{p, \lambda} .
\end{aligned}
$$

The theory of boundedness of classical operators of the real analysis, such as the maximal operator, fractional maximal operator, Riesz potential and the singular integral operators etc., from one weighted Lebesgue space to another one is well studied. Let $f \in L_{1}^{\text {loc }}\left(\mathbb{R}^{n}\right)$. The fractional maximal operator $M_{\alpha}$ and the Riesz potential $I_{\alpha}$ are defined by

$$
\begin{aligned}
& M_{\alpha} f(x)=\sup _{t>0}|B(x, t)|^{-1+\frac{\alpha}{n}} \int_{B(x, t)}|f(y)| d y, \quad 0 \leq \alpha<n, \\
& I_{\alpha} f(x)=\int_{\mathbb{R}^{n}} \frac{f(y) d y}{|x-y|^{n-\alpha}}, \quad 0<\alpha<n .
\end{aligned}
$$

If $\alpha=0$, then $M \equiv M_{0}$ is the Hardy-Littlewood maximal operator. In [2], Chiarenza and Frasca obtained the boundedness of $M$ on $M_{p, \lambda}\left(\mathbb{R}^{n}\right)$. In [3], Adams established the boundedness of $I_{\alpha}$ on $M_{p, \lambda}\left(\mathbb{R}^{n}\right)$.

Here and subsequently, $C$ will denote a positive constant which may vary from line to line but will remain independent of the relevant quantities.

The Calderón-Zygmund singular integral operator is defined by

$$
\widetilde{T} f(x)=p \cdot v \cdot \int_{\mathbb{R}^{n}} K(x-y) f(y) d y,
$$

where $K$ is a Calderón-Zygmund kernel (CZK). We say a kernel $K \in C^{1}\left(\mathbb{R}^{n} \backslash\{0\}\right)$ is a CZK if it satisfies

$$
\begin{aligned}
& |K(x)| \leq \frac{C}{|x|^{n}}, \\
& |\nabla K(x)| \leq \frac{C}{|x|^{n+1}}
\end{aligned}
$$

and

$$
\int_{a<|x|<b} K(x) d x=0
$$

for all $a, b$ with $0<a<b$. Chiarenza and Frasca [2] showed the boundedness of $\widetilde{T}$ on $M_{p, \lambda}\left(\mathbb{R}^{n}\right)$.

It is worth pointing out that the kernel in (1.1) is convolution kernel. However, there were many kinds of operators with non-convolution kernels, such as Fourier transform 
and Radon transform [4], which both are versions of oscillatory integrals. The object we consider in this paper is a class of oscillatory integrals due to Ricci and Stein [5]

$$
T f(x)=p \cdot v \cdot \int_{\mathbb{R}^{n}} e^{i P(x, y)} K(x-y) f(y) d y,
$$

where $P(x, y)$ is a real valued polynomial defined on $\mathbb{R}^{n} \times \mathbb{R}^{n}$, and $K$ is a CZK.

It is well known that the oscillatory factor $e^{i P(x, y)}$ makes it impossible to establish the $L_{p}$ norm inequalities of (1.5) by the method as in the case of Calderón-Zygmund operators or fractional integrals. In [6], Chanillo and Christ established the weak $(1,1)$ type estimate of $T$.

A distribution kernel $K$ is called a standard Calderón-Zygmund kernel (SCZK) if it satisfies the following hypotheses:

$$
|K(x, y)| \leq \frac{C}{|x-y|^{n}}, \quad x \neq y
$$

and

$$
\left|\nabla_{x} K(x, y)\right|+\left|\nabla_{y} K(x, y)\right| \leq \frac{C}{|x-y|^{n+1}}, \quad x \neq y .
$$

The corresponding Calderón-Zygmund integral operator $\widetilde{S}$ and oscillatory integral operator $S$ are defined by

$$
\widetilde{S} f(x)=p \cdot v \cdot \int_{\mathbb{R}^{n}} K(x, y) f(y) d y
$$

and

$$
S f(x)=p \cdot v \cdot \int_{\mathbb{R}^{n}} e^{i P(x, y)} K(x, y) f(y) d y,
$$

where $P(x, y)$ is a real valued polynomial defined on $\mathbb{R}^{n} \times \mathbb{R}^{n}$. In [7], Lu and Zhang proved that $S$ is bounded on $L_{p}$ with $1<p<\infty$. In [5], Ricci and Stein also introduced the standard fractional Calderón-Zygmund kernel (SFCZK) $K_{\alpha}$ with $0<\alpha<n$, where the conditions (1.6) and (1.7) were replaced by

$$
\left|K_{\alpha}(x, y)\right| \leq \frac{C}{|x-y|^{n-\alpha}}, \quad x \neq y
$$

and

$$
\left|\nabla_{x} K_{\alpha}(x, y)\right|+\left|\nabla_{y} K_{\alpha}(x, y)\right| \leq \frac{C}{|x-y|^{n+1-\alpha}}, \quad x \neq y .
$$

The corresponding fractional oscillatory integral operator is defined by (see [8])

$$
S_{\alpha} f(x)=\int_{\mathbb{R}^{n}} e^{i P(x, y)} K_{\alpha}(x, y) f(y) d y
$$

where $P(x, y)$ is also a real valued polynomial defined on $\mathbb{R}^{n} \times \mathbb{R}^{n}$. Obviously, when $\alpha=0$, $S_{0}=S$ and $K_{0}=K$. Partly motivated by the idea from $[9,10]$ and the results of [11], we now give the results of this paper in the following. 
Theorem 1.1 Let $1 \leq p<\infty$, and $\left(\varphi_{1}, \varphi_{2}\right)$ satisfies the condition

$$
\int_{r}^{\infty} \frac{\operatorname{ess}^{\sup _{t<s<\infty}} \varphi_{1}(x, s) s^{\frac{n}{p}}}{t^{\frac{n}{p}+1}} d t \leq C \varphi_{2}(x, r)
$$

where $C$ does not depend on $x$ and $t$. If $K$ is a SCZK and the operator $\widetilde{S}$ is of type $\left(L_{2}\left(\mathbb{R}^{n}\right), L_{2}\left(\mathbb{R}^{n}\right)\right)$, then for $1<p<\infty$ and any polynomial $P(x, y)$ the operator $S$ is bounded from $M_{p, \varphi_{1}}$ to $M_{p, \varphi_{2}}$.

Moreover, for $p=1$ and $K$ is a CZK operator, the operator $T$ is bounded from $M_{1, \varphi_{1}}$ to $W M_{1, \varphi_{2}}$.

Theorem 1.2 Let $1 \leq p<\infty, 0<\alpha<\frac{n}{p}, \frac{1}{q}=\frac{1}{p}-\frac{\alpha}{n}, P(x, y)$ is a polynomial, and $\left(\varphi_{1}, \varphi_{2}\right)$ satisfies the condition

$$
\int_{r}^{\infty} \frac{\operatorname{ess} \sup _{t<s<\infty} \varphi_{1}(x, s) s^{\frac{n}{p}}}{t^{\frac{n}{q}+1}} d t \leq C \varphi_{2}(x, r)
$$

where $C$ does not depend on $x$ and $t$. Then for $p>1$ the operator $S_{\alpha}$ is bounded from $M_{p, \varphi_{1}}$ to $M_{q, \varphi_{2}}$ and for $p=1$ the operator $S_{\alpha}$ is bounded from $M_{1, \varphi_{1}}$ to $W M_{q, \varphi_{2}}$.

For a locally integrable function $b$, the commutator operator formed by $S$ (or $S_{\alpha}$ ) and $b$ are defined by

$$
S_{b} f(x)=b(x) S f(x)-S(b f)(x)
$$

and

$$
S_{\alpha, b} f(x)=b(x) S_{\alpha} f(x)-S_{\alpha}(b f)(x) .
$$

Theorem 1.3 Let $1<p<\infty, b \in \mathrm{BMO}\left(\mathbb{R}^{n}\right)$ and $\left(\varphi_{1}, \varphi_{2}\right)$ satisfies the condition

$$
\int_{r}^{\infty}\left(1+\ln \frac{t}{r}\right) \frac{\operatorname{ess} \sup _{t<s<\infty} \varphi_{1}(x, s) s^{\frac{n}{p}}}{t^{\frac{n}{p}+1}} d t \leq C \varphi_{2}(x, r)
$$

where $C$ does not depend on $x$ and $t$. If $K$ is a SCZK and the operator $\widetilde{S}$ is of type $\left(L_{2}\left(\mathbb{R}^{n}\right), L_{2}\left(\mathbb{R}^{n}\right)\right)$, then for any polynomial $P(x, y)$ the operator $S_{b}$ is bounded from $M_{p, \varphi_{1}}$ to $M_{p, \varphi_{2}}$.

Theorem 1.4 Let $1<p<\infty, b \in \mathrm{BMO}\left(\mathbb{R}^{n}\right), 0<\alpha<\frac{n}{p}, \frac{1}{q}=\frac{1}{p}-\frac{\alpha}{n}, P(x, y)$ is a polynomial, and $\left(\varphi_{1}, \varphi_{2}\right)$ satisfies the condition

$$
\int_{r}^{\infty}\left(1+\ln \frac{t}{r}\right) \frac{\operatorname{ess}^{\sup _{t<s<\infty}} \varphi_{1}(x, s) s^{\frac{n}{p}}}{t^{\frac{n}{q}+1}} d t \leq C \varphi_{2}(x, r)
$$

where $C$ does not depend on $x$ and $t$. Then the operator $S_{b, \alpha}$ is bounded from $M_{p, \varphi_{1}}$ to $M_{q, \varphi_{2}}$. 


\section{Some known results in generalized Morrey spaces $M_{p, \varphi}\left(\mathbb{R}^{n}\right)$}

In $[9,10,12,13]$ and [14], there were obtained sufficient conditions on weights $\varphi_{1}$ and $\varphi_{2}$ for the boundedness of the singular operator $T$ from $\mathcal{M}_{p, \varphi_{1}}\left(\mathbb{R}^{n}\right)$ to $\mathcal{M}_{p, \varphi_{2}}\left(\mathbb{R}^{n}\right)$.

The following statements were proved by Nakai [14].

Theorem A Let $1 \leq p<\infty$ and $\varphi(x, r)$ satisfy the conditions

$$
c^{-1} \varphi(x, r) \leq \varphi(x, t) \leq c \varphi(x, r)
$$

whenever $r \leq t \leq 2 r$, where $c(\geq 1)$ does not depend on $t, r$ and $x \in \mathbb{R}^{n}$ and

$$
\int_{r}^{\infty} \varphi(x, t)^{p} \frac{d t}{t} \leq C \varphi(x, r)^{p}
$$

where $C$ does not depend on $x$ and $r$. Then for $p>1$ the operators $M$ and $T$ are bounded in $\mathcal{M}_{p, \varphi}\left(\mathbb{R}^{n}\right)$ and for $p=1, M$ and $T$ are bounded from $\mathcal{M}_{1, \varphi}\left(\mathbb{R}^{n}\right)$ to $W \mathcal{M}_{1, \varphi}\left(\mathbb{R}^{n}\right)$.

Theorem B Let $1 \leq p<\infty, 0<\alpha<\frac{n}{p}, \frac{1}{q}=\frac{1}{p}-\frac{\alpha}{n}$ and $\varphi(x, t)$ satisfy the conditions (2.1) and

$$
\int_{r}^{\infty} \varphi(x, t)^{p} \frac{d t}{t} \leq C \varphi(x, r)^{p}
$$

where $C$ does not depend on $x$ and $r$. Then for $p>1$, the operators $M_{\alpha}$ and $I_{\alpha}$ are bounded from $\mathcal{M}_{p, \varphi}\left(\mathbb{R}^{n}\right)$ to $\mathcal{M}_{q, \varphi}\left(\mathbb{R}^{n}\right)$ and for $p=1, M_{\alpha}$ and $I_{\alpha}$ are bounded from $\mathcal{M}_{1, \varphi}\left(\mathbb{R}^{n}\right)$ to $W \mathcal{M}_{q, \varphi}\left(\mathbb{R}^{n}\right)$

The following statements, containing Nakai results obtained in $[13,14]$ was proved by Guliyev in $[9,10]$ (see also $[15,16])$.

Theorem $\mathbf{C}$ Let $1 \leq p<\infty$ and $\left(\varphi_{1}, \varphi_{2}\right)$ satisfy the condition

$$
\int_{t}^{\infty} \varphi_{1}(x, r) \frac{d r}{r} \leq C \varphi_{2}(x, t)
$$

where $C$ does not depend on $x$ and $t$. Then the operators $M$ and $T$ are bounded from $M_{p, \varphi_{1}}$ to $M_{p, \varphi_{2}}$ for $p>1$ and from $M_{1, \varphi_{1}}$ to $W M_{1, \varphi_{2}}$.

Theorem D Let $1 \leq p<\infty, 0<\alpha<\frac{n}{p}, \frac{1}{q}=\frac{1}{p}-\frac{\alpha}{n}$ and $\left(\varphi_{1}, \varphi_{2}\right)$ satisfy the condition

$$
\int_{r}^{\infty} t^{\alpha} \varphi_{1}(x, t) \frac{d t}{t} \leq C \varphi_{2}(x, r)
$$

where $C$ does not depend on $x$ and $r$. Then the operators $M_{\alpha}$ and $I_{\alpha}$ are bounded from $M_{p, \varphi_{1}}$ to $M_{q, \varphi_{2}}$ for $p>1$ and from $M_{1, \varphi_{1}}$ to $W M_{q, \varphi_{2}}$ for $p=1$.

The following statements, containing Guliyev results obtained in $[9,10]$ was proved by Guliyev et al. in [11, 12].

Theorem E Let $1 \leq p<\infty$ and $\left(\varphi_{1}, \varphi_{2}\right)$ satisfy the condition (2.4). Then the operators $M$ and $T$ are bounded from $M_{p, \varphi_{1}}$ to $M_{p, \varphi_{2}}$ for $p>1$ and from $M_{1, \varphi_{1}}$ to $W M_{1, \varphi_{2}}$. 
Theorem F Let $1 \leq p<\infty, 0<\alpha<\frac{n}{p}, \frac{1}{q}=\frac{1}{p}-\frac{\alpha}{n}$ and $\left(\varphi_{1}, \varphi_{2}\right)$ satisfy the condition (1.14). Then the operators $M_{\alpha}$ and $I_{\alpha}$ are bounded from $M_{p, \varphi_{1}}$ to $M_{q, \varphi_{2}}$ for $p>1$ and from $M_{1, \varphi_{1}}$ to $W M_{q, \varphi_{2}}$ for $p=1$.

Note that integral conditions of type (2.3) after the paper [17] of 1956 are often referred to as Bary-Stechkin or Zygmund-Bary-Stechkin conditions; see also [18]. The classes of almost monotonic functions satisfying such integral conditions were later studied in a number of papers, see [19-21] and references therein, where the characterization of integral inequalities of such a kind was given in terms of certain lower and upper indices known as Matuszewska-Orlicz indices. Note that in the cited papers the integral inequalities were studied as $r \rightarrow 0$. Such inequalities are also of interest when they allow to impose different conditions as $r \rightarrow 0$ and $r \rightarrow \infty$; such a case was dealt with in [22, 23].

\section{The fractional oscillatory integral operators in the spaces $M_{p, \varphi}\left(\mathbb{R}^{n}\right)$}

In this section, we are going to use the following statement on the boundedness of the Hardy operator:

$$
(H g)(t):=\frac{1}{t} \int_{0}^{t} g(r) d r, \quad 0<t<\infty .
$$

Theorem G [24] The inequality

$$
\underset{t>0}{\operatorname{ess} \sup } w(t) H g(t) \leq c \underset{t>0}{\operatorname{ess} \sup } v(t) g(t)
$$

holds for all non-negative and non-increasing $g$ on $(0, \infty)$ if and only if

$$
A:=\sup _{t>0} \frac{w(t)}{t} \int_{0}^{t} \frac{d r}{\operatorname{essinf}_{0<s<r} v(s)}<\infty,
$$

and $c \approx A$.

Lemma 3.1 Let $1 \leq p<\infty$, and $K$ is a SCZK and the Calderón-Zygmund singular integral operator $\widetilde{S}$ is of type $\left(L_{2}\left(\mathbb{R}^{n}\right), L_{2}\left(\mathbb{R}^{n}\right)\right)$. Then for $1<p<\infty$ and any polynomial $P(x, y)$ the inequality

$$
\|S f\|_{L_{p}\left(B\left(x_{0}, r\right)\right)} \lesssim r^{\frac{n}{p}} \int_{2 r}^{\infty}\|f\|_{L_{p}\left(B\left(x_{0}, t\right)\right.} t^{-1-\frac{n}{p}} d t
$$

holds for any ball $B\left(x_{0}, r\right)$ and for all $f \in L_{p}^{\text {loc }}\left(\mathbb{R}^{n}\right)$.

Moreover, for $p=1$ and $K$ is a $C Z K$

$$
\|T f\|_{W L_{1}\left(B\left(x_{0}, r\right)\right)} \lesssim r^{n} \int_{2 r}^{\infty}\|f\|_{L_{p}\left(B\left(x_{0}, t\right)\right.} t^{-1-n} d t
$$

holds for any ball $B\left(x_{0}, r\right)$ and for all $f \in L_{1}^{\text {loc }}\left(\mathbb{R}^{n}\right)$.

Proof Let $p \in(1, \infty)$. For arbitrary $x_{0} \in \mathbb{R}^{n}$, set $B=B\left(x_{0}, r\right)$ for the ball centered at $x_{0}$ and radius $r, 2 B=B\left(x_{0}, 2 r\right)$. We represent $f$ as

$$
f=f_{1}+f_{2}, \quad f_{1}(y)=f(y) \chi_{2 B}(y), f_{2}(y)=f(y) \chi_{(2 B)} \mathrm{C}(y)
$$


and have

$$
\|S f\|_{L_{p}(B)} \leq\left\|S f_{1}\right\|_{L_{p}(B)}+\left\|S f_{2}\right\|_{L_{p}(B)} .
$$

It is known that (see [5], see also [7, 25, 26]), if $K$ is a SCZK and the operator $\widetilde{S}$ is of type $\left(L_{2}\left(\mathbb{R}^{n}\right), L_{2}\left(\mathbb{R}^{n}\right)\right)$, then for $1<p<\infty$ and any polynomial $P(x, y)$ the operator $S$ is bounded on $L_{p}\left(\mathbb{R}^{n}\right)$. Since $f_{1} \in L_{p}\left(\mathbb{R}^{n}\right), S f_{1} \in L_{p}\left(\mathbb{R}^{n}\right)$ and boundedness of $S$ in $L_{p}\left(\mathbb{R}^{n}\right)$ (see [5]) it follows that

$$
\left\|S f_{1}\right\|_{L_{p}(B)} \leq\left\|S f_{1}\right\|_{L_{p}\left(\mathbb{R}^{n}\right)} \leq C\left\|f_{1}\right\|_{L_{p}\left(\mathbb{R}^{n}\right)}=C\left\|f_{1}\right\|_{L_{p}(2 B)}
$$

where constant $C>0$ is independent of $f$.

It is clear that $x \in B, y \in(2 B)^{\complement}$ implies $\frac{1}{2}\left|x_{0}-y\right| \leq|x-y| \leq \frac{3}{2}\left|x_{0}-y\right|$. We get

$$
\left|S f_{2}(x)\right| \leq c_{0} \int_{(2 B) \mathrm{C}} \frac{|f(y)|}{\left|x_{0}-y\right|^{n}} d y .
$$

By Fubini's theorem and applying Hölder inequality, we have

$$
\begin{aligned}
\int_{(2 B)} \frac{|f(y)|}{\left|x_{0}-y\right|^{n}} d y & \approx \int_{(2 B) \mathrm{C}}|f(y)| \int_{\left|x_{0}-y\right|}^{\infty} t^{-1-n} d t d y \\
& \approx \int_{2 r}^{\infty} \int_{2 r<\left|x_{0}-y\right|<t}|f(y)| d y t^{-1-n} d t \\
& \lesssim \int_{2 r}^{\infty} \int_{B\left(x_{0}, t\right)}|f(y)| d y t^{-1-n} d t \\
& \lesssim \int_{2 r}^{\infty}\|f\|_{L_{p}\left(B\left(x_{0}, t\right)\right.} t^{-1-\frac{n}{p}} d t .
\end{aligned}
$$

Moreover, for all $p \in[1, \infty)$ the inequality

$$
\left\|S f_{2}\right\|_{L_{p}(B)} \lesssim r^{\frac{n}{p}} \int_{2 r}^{\infty}\|f\|_{L_{p}\left(B\left(x_{0}, t\right)\right)} t^{-1-\frac{n}{p}} d t
$$

is valid. Thus,

$$
\|S f\|_{L_{p}(B)} \lesssim\|f\|_{L_{p}(2 B)}+r^{\frac{n}{p}} \int_{2 r}^{\infty}\|f\|_{L_{p}\left(B\left(x_{0}, t\right)\right.} t^{-1-\frac{n}{p}} d t
$$

On the other hand,

$$
\begin{aligned}
\|f\|_{L_{p}(2 B)} & \approx r^{\frac{n}{p}}\|f\|_{L_{p}(2 B)} \int_{2 r}^{\infty} t^{-1-\frac{n}{p}} d t \\
& \lesssim r^{\frac{n}{p}} \int_{2 r}^{\infty}\|f\|_{L_{p}\left(B\left(x_{0}, t\right)\right)} t^{-1-\frac{n}{p}} d t .
\end{aligned}
$$

Hence,

$$
\|S f\|_{L_{p}(B)} \lesssim r^{\frac{n}{p}} \int_{2 r}^{\infty}\|f\|_{L_{p}\left(B\left(x_{0}, t\right)\right)} t^{-1-\frac{n}{p}} d t
$$


Let $p=1$. From the weak $(1,1)$ boundedness of $T$ (see [6]) and (3.4), it follows that:

$$
\begin{aligned}
\left\|T f_{1}\right\|_{W L_{1}(B)} & \leq\left\|T f_{1}\right\|_{W L_{1}\left(\mathbb{R}^{n}\right)} \lesssim\left\|f_{1}\right\|_{L_{1}\left(\mathbb{R}^{n}\right)} \\
& =\|f\|_{L_{1}(2 B)} \lesssim r^{n} \int_{2 r}^{\infty} \int_{B\left(x_{0}, t\right)}|f(y)| d y \frac{d t}{t^{n+1}} .
\end{aligned}
$$

Then by (3.4) and (3.5), we get the inequality (3.1).

Proof of Theorem 1.1 By Lemma 3.1 and Theorem G, we get

$$
\begin{aligned}
\|S f\|_{M_{p, \varphi_{2}}} & \lesssim \sup _{x \in \mathbb{R}^{n}, r>0} \varphi_{2}(x, r)^{-1} \int_{r}^{\infty}\|f\|_{L_{p}(B(x, t))} t^{-1-\frac{n}{p}} d t \\
& \approx \sup _{x \in \mathbb{R}^{n}, r>0} \varphi_{2}(x, r)^{-1} \int_{0}^{r^{-\frac{n}{p}}}\|f\|_{L_{p}\left(B\left(x, t^{-\frac{p}{n}}\right)\right)} d t \\
& =\sup _{x \in \mathbb{R}^{n}, r>0} \varphi_{2}\left(x, r^{-\frac{p}{n}}\right)^{-1} \int_{0}^{r}\|f\|_{L_{p}\left(B\left(x, t^{-\frac{p}{n}}\right)\right)} d t \\
& \lesssim \sup _{x \in \mathbb{R}^{n}, r>0} \varphi_{1}\left(x, r^{-\frac{p}{n}}\right)^{-1} r\|f\|_{L_{p}\left(B\left(x, r^{-} \frac{p}{n}\right)\right)}=\|f\|_{M_{p, \varphi_{1}}}
\end{aligned}
$$

if $p \in(1, \infty)$, and

$$
\begin{aligned}
\|T f\|_{W M_{1, \varphi_{2}}} & \lesssim \sup _{x \in \mathbb{R}^{n}, r>0} \varphi_{2}(x, r)^{-1} \int_{r}^{\infty}\|f\|_{L_{1}(B(x, t))} t^{-1-n} d t \\
& \approx \sup _{x \in \mathbb{R}^{n}, r>0} \varphi_{2}(x, r)^{-1} \int_{0}^{r^{-n}}\|f\|_{L_{1}\left(B\left(x, t^{-\frac{1}{n}}\right)\right)} d t \\
& =\sup _{x \in \mathbb{R}^{n}, r>0} \varphi_{2}\left(x, r^{-\frac{1}{n}}\right)^{-1} \int_{0}^{r}\|f\|_{L_{1}\left(B\left(x, t^{-\frac{1}{n}}\right)\right)} d t \\
& \lesssim \sup _{x \in \mathbb{R}^{n}, r>0} \varphi_{1}\left(x, r^{-\frac{1}{n}}\right)^{-1} r\|f\|_{L_{1}\left(B\left(x, r^{-\frac{1}{n}}\right)\right)}=\|f\|_{M_{1, \varphi_{1}}}
\end{aligned}
$$

if $p=1$.

Proof of Theorem 1.2 The proof of Theorem 1.2 follows from Theorem F and the following observation:

$$
\left|S_{\alpha} f(x)\right| \leq I_{\alpha}(|f|)(x)
$$

\section{Commutators of fractional oscillatory integral operators in the spaces $M_{p, \varphi}\left(\mathbb{R}^{n}\right)$}

Let $T$ be a Calderón-Zygmund singular integral operator and $b \in \mathrm{BMO}\left(\mathbb{R}^{n}\right)$. A well known result of Coifman, Rochberg and Weiss [27] states that the commutator operator $[b, T] f=$ $T(b f)-b T f$ is bounded on $L_{p}\left(\mathbb{R}^{n}\right)$ for $1<p<\infty$. The commutator of Calderón-Zygmund operators plays an important role in studying the regularity of solutions of elliptic partial differential equations of second order (see, for example, [2, 28, 29]).

First, we recall the definition of the space $\mathrm{BMO}\left(\mathbb{R}^{n}\right)$. 
Definition 2 Suppose that $f \in L_{1}^{\text {loc }}\left(\mathbb{R}^{n}\right)$, let

$$
\|f\|_{*}=\sup _{x \in \mathbb{R}^{n}, r>0} \frac{1}{|B(x, r)|} \int_{B(x, r)}\left|f(y)-f_{B(x, r)}\right| d y<\infty,
$$

where

$$
f_{B(x, r)}=\frac{1}{|B(x, r)|} \int_{B(x, r)} f(y) d y .
$$

Define

$$
\operatorname{BMO}\left(\mathbb{R}^{n}\right)=\left\{f \in L_{1}^{\mathrm{loc}}\left(\mathbb{R}^{n}\right):\|f\|_{*}<\infty\right\}
$$

If one regards two functions whose difference is a constant as one, then space $\mathrm{BMO}\left(\mathbb{R}^{n}\right)$ is a Banach space with respect to norm $\|\cdot\|_{*}$.

Remark 1 (1) The John-Nirenberg inequality: there are constants $C_{1}, C_{2}>0$, such that for all $f \in \mathrm{BMO}\left(\mathbb{R}^{n}\right)$ and $\beta>0$

$$
\left|\left\{x \in B:\left|f(x)-f_{B}\right|>\beta\right\}\right| \leq C_{1}|B| e^{-C_{2} \beta /\|f\|_{*}}, \quad \forall B \subset \mathbb{R}^{n} .
$$

(2) The John-Nirenberg inequality implies that

$$
\|f\|_{*} \approx \sup _{x \in \mathbb{R}^{n}, r>0}\left(\frac{1}{|B(x, r)|} \int_{B(x, r)}\left|f(y)-f_{B(x, r)}\right|^{p} d y\right)^{\frac{1}{p}}
$$

for $1<p<\infty$.

(3) Let $f \in \mathrm{BMO}\left(\mathbb{R}^{n}\right)$. Then there is a constant $C>0$ such that

$$
\left|f_{B(x, r)}-f_{B(x, t)}\right| \leq C\|f\|_{*} \ln \frac{t}{r} \quad \text { for } 0<2 r<t
$$

where $C$ is independent of $f, x, r$ and $t$.

Lemma 4.1 Let $1 \leq p<\infty, b \in \mathrm{BMO}\left(\mathbb{R}^{n}\right), K$ is a SCZK and the Calderón-Zygmund singular integral operator $\widetilde{S}$ is of type $\left(L_{2}\left(\mathbb{R}^{n}\right), L_{2}\left(\mathbb{R}^{n}\right)\right)$. Then for $1<p<\infty$ and any polynomial $P(x, y)$ the inequality

$$
\left\|S_{b} f\right\|_{L_{p}\left(B\left(x_{0}, r\right)\right)} \lesssim\|b\|_{*} r^{\frac{n}{p}} \int_{2 r}^{\infty}\|f\|_{L_{p}\left(B\left(x_{0}, t\right)\right)} t^{-1-\frac{n}{p}} d t
$$

holds for any ball $B\left(x_{0}, r\right)$ and for all $f \in L_{p}^{\text {loc }}\left(\mathbb{R}^{n}\right)$.

Proof Let $p \in(1, \infty)$. For arbitrary $x_{0} \in \mathbb{R}^{n}$, set $B=B\left(x_{0}, r\right)$ for the ball centered at $x_{0}$ and radius $r, 2 B=B\left(x_{0}, 2 r\right)$. We represent $f$ as

$$
f=f_{1}+f_{2}, \quad f_{1}(y)=f(y) \chi_{2 B}(y), f_{2}(y)=f(y) \chi_{(2 B)} \mathrm{C}(y)
$$


and have

$$
\left\|S_{b} f\right\|_{L_{p}(B)} \leq\left\|S_{b} f_{1}\right\|_{L_{p}(B)}+\left\|S_{b} f_{2}\right\|_{L_{p}(B)} .
$$

It is known that (see [5], see also [7, 25, 26]), if $K$ is a SCZK and the operator $\widetilde{S}$ is of type $\left(L_{2}\left(\mathbb{R}^{n}\right), L_{2}\left(\mathbb{R}^{n}\right)\right)$, then for $1<p<\infty$ and any polynomial $P(x, y)$ the commutator operator $S_{b}$ is bounded on $L_{p}\left(\mathbb{R}^{n}\right)$. Since $f_{1} \in L_{p}\left(\mathbb{R}^{n}\right), S f_{1} \in L_{p}\left(\mathbb{R}^{n}\right)$ and boundedness of $S_{b}$ in $L_{p}\left(\mathbb{R}^{n}\right)$ (see [5]) it follows that

$$
\left\|S_{b} f_{1}\right\|_{L_{p}(B)} \leq\left\|S_{b} f_{1}\right\|_{L_{p}\left(\mathbb{R}^{n}\right)} \leq C\|b\|_{*}\left\|f_{1}\right\|_{L_{p}\left(\mathbb{R}^{n}\right)}=C\|b\|_{*}\left\|f_{1}\right\|_{L_{p}(2 B)},
$$

where constant $C>0$ is independent of $f$.

For $x \in B$, we have

$$
\begin{aligned}
\left|S_{b} f_{2}(x)\right| & \lesssim \int_{\mathbb{R}^{n}} \frac{|b(y)-b(x)|}{|x-y|^{n}}|f(y)| d y \\
& \approx \int_{\mathrm{C}_{(2 B)}} \frac{|b(y)-b(x)|}{\left|x_{0}-y\right|^{n}}|f(y)| d y .
\end{aligned}
$$

Then

$$
\begin{aligned}
\left\|S_{b} f_{2}\right\|_{L_{p}(B)} \lesssim & \left(\int_{B}\left(\int_{\mathrm{C}_{(2 B)}} \frac{|b(y)-b(x)|}{\left|x_{0}-y\right|^{n}}|f(y)| d y\right)^{p} d x\right)^{\frac{1}{p}} \\
\lesssim & \left(\int_{B}\left(\int_{\mathrm{C}_{(2 B)}} \frac{\left|b(y)-b_{B}\right|}{\left|x_{0}-y\right|^{n}}|f(y)| d y\right)^{p} d x\right)^{\frac{1}{p}} \\
& +\left(\int_{B}\left(\int_{\mathrm{C}_{(2 B)}} \frac{\left|b(x)-b_{B}\right|}{\left|x_{0}-y\right|^{n}}|f(y)| d y\right)^{p} d x\right)^{\frac{1}{p}} \\
= & I_{1}+I_{2} .
\end{aligned}
$$

Let us estimate $I_{1}$.

$$
\begin{aligned}
I_{1} & \approx r^{\frac{n}{p}} \int_{\mathrm{C}_{(2 B)}} \frac{\left|b(y)-b_{B}\right|}{\left|x_{0}-y\right|^{n}}|f(y)| d y \\
& \approx r^{\frac{n}{p}} \int_{\mathrm{C}_{(2 B)}}\left|b(y)-b_{B}\right||f(y)| \int_{\left|x_{0}-y\right|}^{\infty} \frac{d t}{t^{n+1}} d y \\
& \approx r^{\frac{n}{p}} \int_{2 r}^{\infty} \int_{2 r \leq\left|x_{0}-y\right| \leq t}\left|b(y)-b_{B}\right||f(y)| d y \frac{d t}{t^{n+1}} \\
& \lesssim r^{\frac{n}{p}} \int_{2 r}^{\infty} \int_{B\left(x_{0}, t\right)}\left|b(y)-b_{B}\right||f(y)| d y \frac{d t}{t^{n+1}} .
\end{aligned}
$$

Applying Hölder's inequality and by (4.1), (4.2), we get

$$
\begin{aligned}
I_{1} \lesssim r^{\frac{n}{p}} \int_{2 r}^{\infty} \int_{B\left(x_{0}, t\right)}\left|b(y)-b_{B\left(x_{0}, t\right)}\right||f(y)| d y \frac{d t}{t^{n+1}} \\
\quad+r^{\frac{n}{p}} \int_{2 r}^{\infty}\left|b_{B\left(x_{0}, r\right)}-b_{B\left(x_{0}, t\right)}\right| \int_{B\left(x_{0}, t\right)}|f(y)| d y \frac{d t}{t^{n+1}}
\end{aligned}
$$




$$
\begin{aligned}
& \lesssim r^{\frac{n}{p}} \int_{2 r}^{\infty}\left(\int_{B\left(x_{0}, t\right)}\left|b(y)-b_{B\left(x_{0}, t\right)}\right|^{p^{\prime}} d y\right)^{\frac{1}{p^{\prime}}}\|f\|_{L_{p}\left(B\left(x_{0}, t\right)\right)} \frac{d t}{t^{n+1}} \\
& \quad+r^{\frac{n}{p}} \int_{2 r}^{\infty}\left|b_{B\left(x_{0}, r\right)}-b_{B\left(x_{0}, t\right)}\right|\|f\|_{L_{p}\left(B\left(x_{0}, t\right)\right)} t^{-1-\frac{n}{p}} d t \\
& \lesssim\|b\|_{*} r^{\frac{n}{p}} \int_{2 r}^{\infty}\left(1+\ln \frac{t}{r}\right)\|f\|_{L_{p}\left(B\left(x_{0}, t\right)\right)} t^{-1-\frac{n}{p}} d t .
\end{aligned}
$$

In order to estimate $I_{2}$ note that

$$
I_{2}=\left(\int_{B}\left|b(x)-b_{B}\right|^{p} d x\right)^{\frac{1}{p}} \int_{\mathrm{C}_{(2 B)}} \frac{|f(y)|}{\left|x_{0}-y\right|^{n}} d y .
$$

By (4.1), we get

$$
I_{2} \lesssim\|b\|_{*} r^{\frac{n}{p}} \int_{\mathrm{C}_{(2 B)}} \frac{|f(y)|}{\left|x_{0}-y\right|^{n}} d y
$$

Thus, by (3.2)

$$
I_{2} \lesssim\|b\|_{*} r^{\frac{n}{p}} \int_{2 r}^{\infty}\|f\|_{L_{p}\left(B\left(x_{0}, t\right)\right)} t^{-1-\frac{n}{p}} d t
$$

Summing up $I_{1}$ and $I_{2}$, for all $p \in(1, \infty)$ we get

$$
\left\|S_{b} f_{2}\right\|_{L_{p}(B)} \lesssim\|b\|_{*} r^{\frac{n}{p}} \int_{2 r}^{\infty}\left(1+\ln \frac{t}{r}\right)\|f\|_{L_{p}\left(B\left(x_{0}, t\right)\right)} t^{-1-\frac{n}{p}} d t
$$

Finally,

$$
\left\|S_{b} f\right\|_{L_{p}(B)} \lesssim\|b\|_{*}\|f\|_{L_{p}(2 B)}+\|b\|_{*} r^{\frac{n}{p}} \int_{2 r}^{\infty}\left(1+\ln \frac{t}{r}\right)\|f\|_{L_{p}\left(B\left(x_{0}, t\right)\right)} t^{-1-\frac{n}{p}} d t,
$$

and statement of Lemma 4.1 follows by (3.4).

Proof of Theorem 1.3 The statement of Theorem 1.3 follows by Lemma 4.1 and Theorem G in the same manner as in the proof of Theorem G.

Proof of Theorem 1.4 The proof of Theorem 1.4 follows from the Theorem 7.4 in [11] and the following observation:

$$
\left|S_{\alpha, b} f(x)\right| \leq I_{\alpha, b}(|f|)(x)
$$




\section{References}

1. Morrey, CB: On the solutions of quasi-linear elliptic partial differential equations. Trans. Am. Math. Soc. 43, 126-166 (1938)

2. Chiarenza, F, Frasca, M: Morrey spaces and Hardy-Littlewood maximal function. Rend. Mat. Appl. 7, $273-279$ (1987)

3. Adams, DR: A note on Riesz potentials. Duke Math. J. 42, 765-778 (1975)

4. Phong, DH, Stein, EM: Singular integrals related to the Radon transform and boundary value problems. Proc. Natl. Acad. Sci. USA 80, 7697-7701 (1983)

5. Ricci, F, Stein, EM: Harmonic analysis on nilpotent groups and singular integrals I: oscillatory integrals. J. Funct. Anal. 73, 179-194 (1987)

6. Chanillo, S, Christ, M: Weak $(1,1)$ bounds for oscillatory singular integral. Duke Math. J. 55, 141-155 (1987)

7. Lu, SZ, Zhang, Y: Criterion on $L^{P}$-boundedness for a class of oscillatory singular integrals with rough kernels. Rev. Mat. Iberoam. 8, 201-219(1992)

8. Ding, $Y: L_{p}$-Boundedness for fractional oscillatory integral operator with rough kernel. Approx. Theory Appl. 12, 70-79 (1996)

9. Guliyev, VS: Integral operators on function spaces on the homogeneous groups and on domains in $\mathbb{R}^{n}$. Doctor of Sciences, Mat. Inst. Steklova, Moscow (1994), 329 pp. (in Russian)

10. Guliyev, VS: Boundedness of the maximal, potential and singular operators in the generalized Morrey spaces. J. Inequal. Appl. 2009, Article ID 503948 (2009)

11. Guliyev, VS, Aliyev, SS, Karaman, T, Shukurov, PS: Boundedness of sublinear operators and commutators on generalized Morrey space. Integral Equ. Oper. Theory 71, 327-355 (2011)

12. Akbulut, A, Guliyev, VS, Mustafayev, R: On the boundedness of the maximal operator and singular integral operators in generalized Morrey spaces. Math. Bohem. 137(1), 27-43 (2012)

13. Mizuhara, T: Boundedness of some classical operators on generalized Morrey spaces. In: Igari, S (ed.) Harmonic Analysis. ICM 90 Satellite Proceedings, pp. 183-189. Springer, Tokyo (1991)

14. Nakai, E: Hardy-Littlewood maximal operator, singular integral operators and Riesz potentials on generalized Morrey spaces. Math. Nachr. 166, 95-103 (1994)

15. Sawano, Y, Sugano, S, Tanaka, H: A note on generalized fractional integral operators on generalized Morrey spaces. Bound. Value Probl. 2009, Article ID 835865 (2009)

16. Softova, L: Singular integrals and commutators in generalized Morrey spaces. Acta Math. Sin. Engl. Ser. 22(3), 757-766 (2006)

17. Bary, NK, Stechkin, SB: Best approximations and differential properties of two conjugate functions. Tr. Mosk. Mat. Obŝ. 5, 483-522 (1956) (in Russian)

18. Guseinov, Al, Mukhtarov, KS: Introduction to the Theory of Nonlinear Singular Integral Equations. Nauka, Moscow (1980) (in Russian)

19. Karapetiants, NK, Samko, NG: Weighted theorems on fractional integrals in the generalized Hölder spaces $H_{0}^{\omega}(\rho)$ via the indices $m_{\omega}$ and $M_{\omega}$. Fract. Calc. Appl. Anal. 7(4), 437-458 (2004)

20. Samko, N: Singular integral operators in weighted spaces with generalized Hölder condition. Proc. A. Razmadze Math. Inst. 120, 107-134 (1999)

21. Samko, N: On non-equilibrated almost monotonic functions of the Zygmund-Bary-Stechkin class. Real Anal. Exch. 30(2), 727-745 (2004/2005)

22. Kokilashvili, V, Samko, S: Operators of harmonic analysis in weighted spaces with non-standard growth. J. Math. Anal. Appl. 352, 15-34 (2009)

23. Samko, N, Samko, S, Vakulov, B: Weighted Sobolev theorem in Lebesgue spaces with variable exponent. J. Math. Anal. Appl. 335, 560-583 (2007)

24. Carro, M, Pick, L, Soria, J, Stepanov, VD: On embeddings between classical Lorentz spaces. Math. Inequal. Appl. 4(3), 397-428 (2001)

25. Lu, SZ: A class of oscillatory integrals. Int. J. Appl. Math. Sci. 2(1), 42-58 (2005)

26. Lu, SZ, Ding, Y, Yan, DY: Singular Integrals and Related Topics. World Scientific, Singapore (2007)

27. Coifman, R, Rochberg, R, Weiss, G: Factorization theorems for Hardy spaces in several variables. Ann. Math. 103(2), 611-635 (1976)

28. Chiarenza, F, Frasca, M, Longo, P: Interior $W^{2, p}$-estimates for nondivergence elliptic equations with discontinuous coefficients. Ric. Mat. 40, 149-168 (1991)

29. Fazio, GD, Ragusa, MA: Interior estimates in Morrey spaces for strong solutions to nodivergence form equations with discontinuous coefficients. J. Funct. Anal. 112, 241-256 (1993) 